\title{
Eficiência de um sistema piloto utilizando áreas alagadas no pós-tratamento do lixiviado gerado no Aterro Sanitário de Curitiba, Curitiba, Paraná, Brasil
}

\author{
Efficiency of a pilot system using wetlands in the post-treatment of the \\ leachate generated in the Curitiba Landfill, Curitiba, Paraná, Brazil
}

\section{Ana Maria Pereira Barreto Amorim', Thaís Lopes Cavalheiro', Karla Heloise Preussler², Erica Costa Mielke ${ }^{3}$, Selma Aparecida Cubas ${ }^{4}$, Leila Teresinha Maranho ${ }^{5 *}$}

\begin{abstract}
RESUMO
No Aterro Sanitário de Curitiba ocorre o pós-tratamento do lixiviado em áreas alagadas naturais. Para avaliar o papel das macrófitas nesse pós-tratamento, foi desenvolvido um sistema piloto. Dessa forma, o objetivo do presente estudo foi avaliar a eficiência do sistema piloto utilizado para o pós-tratamento do lixiviado desse aterro sanitário. O sistema foi construído com duas caixas dágua: na primeira foi colocada a macrófita Echinochloa polystachya e na segunda, Eichhornia crassipes. O sistema operou em batelada e foi realizado um ciclo de sete dias. Para o monitoramento do sistema foram efetuadas coletas e análises do lixiviado, do substrato e das macrófitas. A eficiência do sistema no pós-tratamento do lixiviado foi de 72\% para demanda bioquímica de oxigênio, 30\% para demanda química de oxigênio, 76\% para fósforo total e fósforo inorgânico, 38\% para nitrato, 33\% para nitrito, 58\% para nitrogênio amonical, 44\% para nitrogênio orgânico e 13\% para zinco.
\end{abstract}

Palavras-chave: fitorremediação; banhados, macrófitas; sistemas simplificados de tratamento

\begin{abstract}
In the Curitiba Landfill the post-treatment of the leachate occurs in natural wetlands. In order to evaluate the role of macrophytes in this post-treatment, it was developed a pilot system. Thus, the objective of this study was to evaluate the effectiveness of the pilot system in the post-treatment of the leachate in this landfill. The system was built with two water tanks: the macrophyte Echinochloa polystachya was placed in the first one; and the Eichhornia crassipes was placed in the second one. The system operated in batch in a seven-day cycle. For monitoring the system, samples were collected, and analyses of the leachate, the substrate, and the macrophytes were performed. The efficiency of the system in the post-treatment of the leachate was $72 \%$ for biochemical oxygen demand, 30\% for chemical oxygen demand, 76\% for total phosphorus and inorganic phosphorus, 38\% for nitrate, 33\% for nitrite, 58\% for ammoniacal nitrogen, $44 \%$ for organic nitrogen, and 13\% for zinc.
\end{abstract}

Keywords: phytoremediation; wetlands; macrophytes; simplified treatment systems.

\section{INTRODUÇÃO}

O lixiviado, devido à alta carga poluidora, passa por sistemas de tratamento que combinam processos físicos, químicos e biológicos antes de ser despejado em corpos hídricos (CASTILHOS et al., 2010). No entanto, a composição complexa e variável do lixiviado (BERTAZZOLI; PELEGRINI, 2002; CELERE et al., 2007; JONES et al., 2006; RENOU et al., 2008), associada ao envelhecimento dos aterros sanitários, reduz a eficiência dos tratamentos (RENOU et al., 2008).
Diante desse fato, é necessário o desenvolvimento de tratamentos alternativos complementares. Nesse contexto, a fitorremediação, em áreas alagadas, se apresenta como uma tecnologia promissora para o tratamento do lixiviado (BRIX, 1994; KADLEC; ZMARTHIE, 2010; MANNARINO et al., 2006; ZHANG et al., 2010). As áreas alagadas são ecossistemas que apresentam alta produtividade primária e papel fundamental nos ciclos biogeoquímicos, características associadas à presença de macrófitas (BRIX, 1994; 1997; CARVALHO; OZORIO, 2007; POMPÊO, 2008).

$\square$

Universidade Positivo - Curitiba (PR), Brasil.

¿Universidade do Rio de Janeiro - Rio de Janeiro (RJ), Brasil.

${ }^{3}$ Diretora do Departamento de Pesquisa e Monitoramento da Secretaria Municipal do Meio Ambiente de Curitiba - Curitiba (PR), Brasil.

${ }^{4}$ Professora titular do curso de Engenharia Civil e do Programa de Pós-Graduação em Gestão Ambiental da Universidade Positivo - Curitiba (PR), Brasil. ${ }^{5}$ Professora titular do curso de Ciências Biológicas e do Programa de Pós-Graduação em Gestão Ambiental da Universidade Positivo - Curitiba (PR), Brasil!

*Autor correspondente: maranho@up.edu.br

Recebido: 20/06/2012 - Aceito: 10/03/2017 - Reg. ABES: 94495 
As macrófitas encontradas em áreas alagadas apresentam diferentes mecanismos, que estão associados à morfofisiologia dos seus tecidos e são adaptados para o desenvolvimento em locais com grande quantidade de água. Em áreas alagadas elas podem controlar a erosão, promover a filtração e superfície para o desenvolvimento de micro-organismos, absorção de nutrientes e liberação de oxigênio (BRIX, 1994; 1997).

O desenvolvimento e a aplicação de um sistema piloto permitem a obtenção de informações sobre o uso de áreas alagadas para o pós-tratamento de lixiviado, além de indicarem o papel das macrófitas nesse processo. Dessa forma, o objetivo do presente estudo foi avaliar a eficiência desse sistema, utilizando áreas alagadas, para o pós-tratamento do lixiviado gerado no Aterro Sanitário de Curitiba.

\section{METODOLOGIA}

O presente estudo foi realizado no Aterro Sanitário da Caximba (Curitiba, Paraná, Brasil), localizado próximo às coordenadas $25^{\circ} 62^{\prime} \mathrm{S}$ e $49^{\circ} 33^{\prime} \mathrm{W}$. A área total do aterro é de $1.015 \mathrm{~km}^{2}$ e a área para disposição de resíduos, de $439,54 \mathrm{~km}^{2}$. O aterro iniciou sua operação em 1989 e foi desativado em outubro de 2010. Desde janeiro de 2011, o lixiviado gerado, depois de tratado por sistema convencional, composto por um desarenador e um conjunto de quatro lagoas, sendo uma primeira de equalização, seguida por duas lagoas aeradas em paralelo e por uma facultativa, segue para um sistema de pós-tratamento constituído por três áreas alagadas e depois é encaminhado para o rio Iguaçu.

Para a construção do sistema piloto foram utilizadas duas caixas d'água, a primeira de $5.000 \mathrm{~L}$ e a segunda, de $3.000 \mathrm{~L}$, nas quais foi colocado, aproximadamente, um volume de $450 \mathrm{~L}$ de lixiviado (Figura 1). O sistema operou em batelada e foi realizado um ciclo de sete dias. Também recebeu o lixiviado proveniente do tratamento convencional adotado no aterro sem diluição.
Na primeira caixa foi colocada, primeiramente, uma camada de $20 \mathrm{~cm}$ de altura de pedra brita 4, seguida de uma camada de $20 \mathrm{~cm}$ de pedra brita 1 e por fim uma camada de $30 \mathrm{~cm}$ de substrato.

As macrófitas empregadas no sistema foram selecionadas por meio de levantamento fitossociológico das áreas alagadas naturais. Para tanto, estabeleceram-se dois transectos (faixas) permanentes posicionados transversalmente e paralelamente ao longo de cada uma das três áreas alagadas naturais. Durante a amostragem, um quadrado de policloreto de vinila (PVC) com dimensão de $1 \mathrm{~m}^{2}$ foi lançado 10 vezes, aleatoriamente, ao longo de cada transecto, totalizando 60 parcelas.

A partir desse levantamento foram selecionadas as três espécies com maior grau de cobertura vegetal, segundo escala proposta por Braun-Blanquet (1979). Posteriormente, essas foram avaliadas quanto à tolerância ao lixiviado, em que 50 exemplares de cada uma foram colocados em contato com ele. Durante um mês foram feitas observações semanais para avaliar o desenvolvimento das macrófitas ante o lixiviado. As duas espécies que apresentaram tolerância ao lixiviado foram coletadas nas áreas alagadas naturais para compor o sistema.

Após coleta, foram selecionados exemplares de Echinochloa polystachya e Eichhornia crassipes. No substrato foram plantadas 50 estacas com 60 a $80 \mathrm{~cm}$ de comprimento de E. polystachya, macrófita que apresenta forma biológica emergente. Após sete dias foi realizada a passagem do lixiviado para a segunda caixa e o esgotamento da primeira. $\mathrm{Na}$ segunda caixa foram colocados 95 exemplares de E. crassipes, espécie que apresenta forma biológica flutuante livre.

Os ciclos de batelada foram realizados durante quatro meses, até estabilização e constatação da sobrevivência, crescimento e desenvolvimento das macrófitas. Após esse período, para avaliar a eficiência do pós-tratamento no sistema piloto efetivaram-se coletas do lixiviado no início (ponto 1), após uma semana na primeira caixa (ponto 2) e no fim do pós-tratamento (ponto 3) após 7 dias de detenção nessa unidade. Os seguintes parâmetros físicos e químicos do lixiviado foram analisados, em triplicata, de

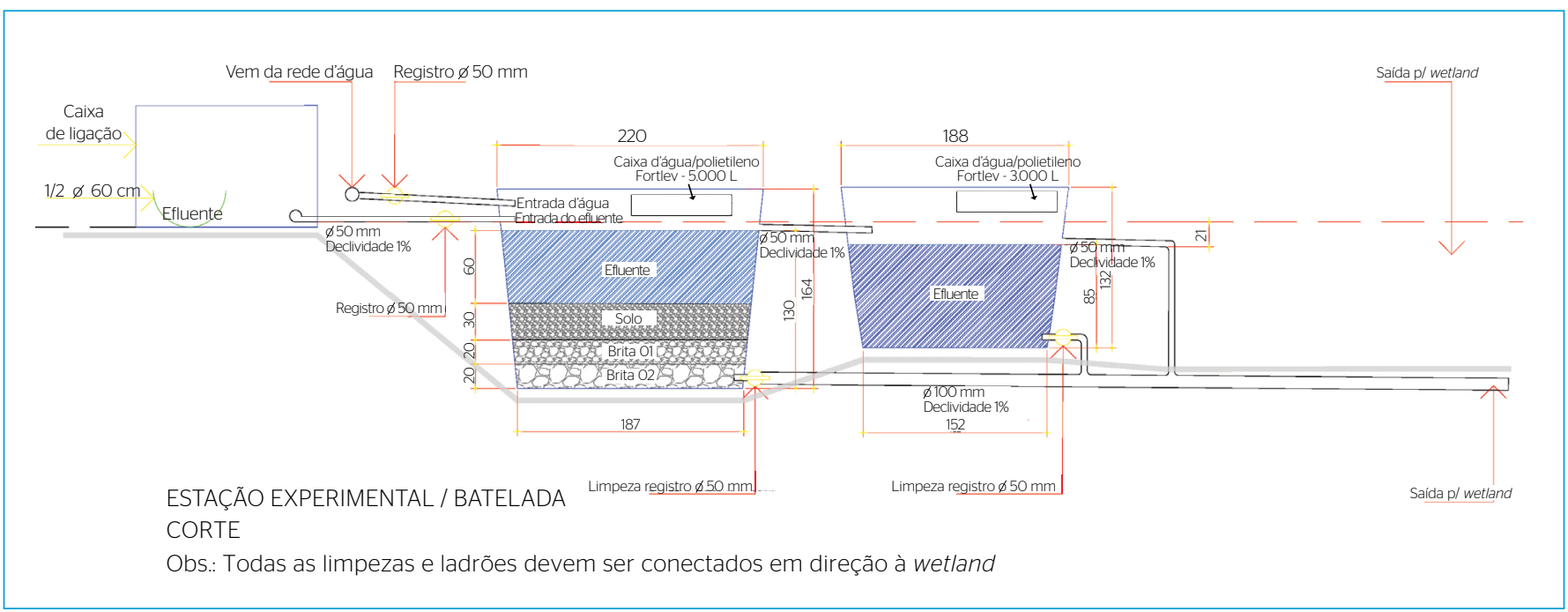

Figura 1 - Esquema do sistema piloto utilizado para o pós-tratamento do lixiviado no Aterro Sanitário da Caximba, Curitiba, Paraná, Brasil. 
acordo com Standard Methods for Examination of Water and Wastewater (APHA; AWWA; WEF, 2005): $\mathrm{pH}$, demanda bioquímica de oxigênio (DBO) $\left(\mathrm{mg} \mathrm{O}_{2} \cdot \mathrm{L}^{-1}\right)$, demanda química de oxigênio (DQO) $\left(\mathrm{mg} \mathrm{O}_{2} \cdot \mathrm{L}^{-1}\right)$, nitrogênio amoniacal $\left(\mathrm{N}^{-\mathrm{NH}_{4}}\right)\left(\mathrm{mg} . \mathrm{L}^{-1}\right)$, nitrogênio orgânico $(\mathrm{N}-\mathrm{NOrg})\left(\mathrm{mg} . \mathrm{L}^{-1}\right)$, nitrito $\left(\mathrm{mg} . \mathrm{L}^{-1}\right)$, nitrato $\left(\mathrm{mg} . \mathrm{L}^{-1}\right)$, fósforo total $(\mathrm{PT})\left(\mathrm{mg} . \mathrm{L}^{-1}\right)$ e fosfato total $\left(\mathrm{P}-\mathrm{PO}_{4}\right)\left(\mathrm{mg} \cdot \mathrm{L}^{-1}\right)$.

Para determinação de metais, a abertura das amostras foi feita em triplicata, segundo método da Companhia Ambiental do Estado de São Paulo (CETESB, 2004). Posteriormente, as amostras foram analisadas quantitativamente para cádmio $(\mathrm{Cd})$, cobre $(\mathrm{Cu})$, níquel $(\mathrm{Ni})$ e zinco $(\mathrm{Zn})$ no espectrofotômetro de absorção atômica (EAA) Shimadzu, modelo AA-6800.

A eficiência do pós-tratamento em termos de $\mathrm{DBO}, \mathrm{DQO}, \mathrm{N}^{-\mathrm{NH}_{4}}$, $\mathrm{N}-\mathrm{NOrg}$, nitrito, nitrato, $\mathrm{PT}, \mathrm{P}-\mathrm{PO}_{4}$ e metais foi calculada segundo Kadlec e Knight (1996). O cálculo da eficiência do sistema piloto foi executado no início do pós-tratamento (ponto 1), em relação ao seu término (ponto 3).

Após o lixiviado ficar uma semana em cada caixa do sistema foram realizadas coletas das macrófitas. Para determinação de metais foram coletados dez exemplares de E. polystachya e de E. crassipes em cada caixa e em uma área de referência (área alagada presentes na área do aterro, mas sem o contato com o lixiviado) que constituiu o controle. A abertura das amostras foi realizada em triplicata, segundo Carneiro et al. (2006) e a análise quantitativa de $\mathrm{Cd}, \mathrm{Cu}, \mathrm{Ni} \mathrm{e} \mathrm{Zn,} \mathrm{no} \mathrm{EAA} \mathrm{Shimadzu,} \mathrm{modelo} \mathrm{AA-6800.}$

Para a dosagem de clorofila foram coletadas seis folhas de seis exemplares das macrófitas de cada caixa e no controle. O teor de clorofila total foi realizado segundo Barnes et al. (1992) e as leituras em espectrofotômetro (UV - Visible Shimadzu, modelo UV1601).

O substrato usado na primeira caixa foi coletado no início do experimento e após uma semana em contato com o lixiviado para determinação de metais. A abertura das amostras foi feita segundo Método 3050 B (U. S. EPA, 1998) e a análise quantitativa de Cd, Cu, Ni e Zn no EAA Shimadzu, modelo AA-6800.

A série temporal das variáveis meteorológicas de precipitação acumulada do dia $(\mathrm{mm})$ e da temperatura do ar $\left({ }^{\circ} \mathrm{C}\right)$ (máxima, média e mínima) da área de estudo foi coletada na estação meteorológica, sob responsabilidade do Instituto Tecnológico SIMEPAR (Sistema Meteorológico do Paraná).

Para a análise da dosagem de clorofila total foi aplicado o teste de MannWhitney. Já para os dados de quantificação de metais das duas espécies de macrófitas estudadas e do substrato, empregou-se o teste $t$ de Student bimodal para amostras independentes e com variâncias aparentemente iguais. Sendo consideradas significativas as diferenças em que $p \leq 0,05$. As análises estatísticas foram realizadas por meio do software BioStat, versão 5.0.

\section{RESULTADOS}

A partir do levantamento fitossociológico, constatou-se que a estrutura da vegetação das áreas alagadas naturais está representada, principalmente, por três espécies (Tabela 1), as quais foram selecionadas para o trabalho: Echinochloa polystachya (Kunth) Hitchc. (capim-cabeludo) com 17\% de cobertura relativa, Pistia stratiotes L. (alface-d’água) com 32\% de cobertura relativa e Eichhornia crassipes (Mart.) Solms (aguapé) com 46\% de cobertura relativa.

As três macrófitas com maior cobertura relativa, $P$. stratiotes, E. polystachya e E. crassipes, ficaram em contato com o lixiviado proveniente do sistema de tratamento convencional sem diluição e, após um mês de observação, os exemplares de $P$. stratiotes não sobreviveram (100\% de morte). Assim, foram escolhidas para compor o sistema E. polystachya e E. crassipes, na primeira e na segunda caixa, respectivamente.

Os resultados dos parâmetros analisados no lixiviado nos três pontos de coleta e a eficiência do pós-tratamento no sistema podem ser consultados na Tabela 2 .

Nas amostras do substrato não foram detectadas concentrações de $\mathrm{Cd}$ e $\mathrm{Cu}$. No início do experimento foram obtidos no substrato os

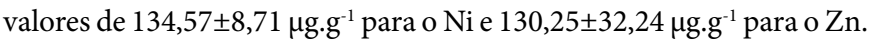
Após uma semana, foram quantificados os valores de 46,00 $\pm 5,18 \mu \mathrm{g} \cdot \mathrm{g}^{-1}$

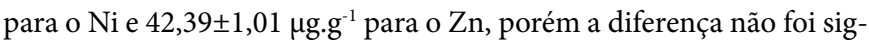
nificativa quando comparados esses valores.

Tabela 1 - Cobertura da macrófitas presentes no sistema de áreas alagadas naturais do Aterro Sanitário da Caximba, Curitiba, Paraná, Brasil.

\begin{tabular}{|c|c|c|c|c|c|c|}
\hline Familia & Espécie & FA (\%) & FR (\%) & $\mathrm{AC}$ & VC & CR (\%) \\
\hline Araceae & Pistia stratiotes L. & 51,67 & 29,78 & 0,17 & 0,28 & 31,54 \\
\hline Araliaceae & Hydrocotyle bonariensis Lam. & 15,00 & 6,52 & 0,01 & 0,03 & 3,23 \\
\hline Orchidaceae & Habenaria repens Nutt. & 10,00 & 4,35 & 0,00 & 0,01 & 0,60 \\
\hline Poacea e & Echinochloa polystachya (Kunth) Hitchc. & 40,00 & 23,05 & 0,09 & 0,16 & 17,17 \\
\hline Pontederiaceae & Eichhornia crassipes (Mart.) Solms & 61,67 & 35,54 & 0,25 & 0,42 & 45,90 \\
\hline Salviniaceae & Salvinia auriculata Aubl. & 25,00 & 10,87 & 0,01 & 0,06 & 5,76 \\
\hline Indeterminada & Espécie indeterminada 1 & 5,00 & 2,17 & 0,00 & 0,02 & 1,69 \\
\hline Indeterminada & Espécie indeterminada 2 & 5,00 & 2,17 & 0,00 & 0,01 & 0,73 \\
\hline Indeterminada & Espécie indeterminada 3 & 5,00 & 2,17 & 0,00 & 0,00 & 0,10 \\
\hline Indeterminada & Espécie indeterminada 4 & 10,00 & 4,35 & 0,00 & 0,01 & 1,19 \\
\hline
\end{tabular}

FA: frequência absoluta; FR: frequência relativa; AC: área de cobertura da espécie; VC: valor de cobertura da espécie; CR: valor de cobertura relativa. 
Ao comparar os teores de clorofila total, para E. polystachya não houve diferença significativa entre as plantas coletadas no sistema e no controle, porém para E. crassipes as plantas coletadas no controle apresentaram o maior teor (Figura 2).

$\mathrm{Na}$ análise de metais houve diferença significativa $(\mathrm{p} \leq 0,05)$ entre as concentrações de Cd e Zn na parte aérea e de Ni nas raízes dos exemplares de E. polystachya do sistema e do controle, sendo as maiores médias as encontradas nos exemplares do sistema. Já na parte aérea e nas raízes desse espécime não foram detectadas concentrações de $\mathrm{Cu}$ (Tabela 3 ).

As concentrações de Cd e Zn na parte aérea e nas raízes de E. crassipes no sistema e no controle apresentaram diferenças significativas $(p \leq 0,05)$, com os maiores valores no sistema. Não houve diferença significativa entre as concentrações de $\mathrm{Cd}$, Ni e $\mathrm{Zn}$ da parte aérea e das raízes de E. crassipes no sistema e no controle (Tabela 3).

Não foi detectado Cu na parte aérea de E. crassipes no controle, no entanto, constatou-se $15,77 \pm 2,13 \mu \mathrm{g} \cdot \mathrm{g}^{-1}$ nas raízes do controle. Já para os exemplares coletados no sistema foram obtidos valores de $15,27 \pm 2,87 \mu \mathrm{g} . \mathrm{g}^{-1}$ nas raízes e de $21,94 \pm 4,86 \mu \mathrm{g} \cdot \mathrm{g}^{-1}$ na parte aérea (Tabela 3 ).

Durante o período em que ocorreu a avaliação do pós-tratamento no sistema, a temperatura média do ar foi $16^{\circ} \mathrm{C}$, com mínima de $9,4^{\circ} \mathrm{C}$ e máxima de $29,1^{\circ} \mathrm{C}$, e a precipitação pluviométrica acumulada total $123 \mathrm{~mm}$.

\section{DISCUSSÃO}

As três espécies com maior área de cobertura, E. polystachya, E. crassipes e $P$. stratiotes apresentam elevada produtividade. Principalmente, em ambientes com grande quantidade de $\mathrm{Ne}$, esses organismos podem crescer excessivamente e prejudicar os ecossistemas aquáticos (POMPÊO, 2008). Em contrapartida, por mostrarem crescimento rápido, essas macrófitas podem ser sugeridas para a fitorremediação, pois a elevada produção de biomassa está relacionada a maiores taxas de remoção e estoque de nutrientes (BARTUCCA et al., 2016; GRANATO, 1995; LAMEGO; VIDAL, 2007; PREUSSLER et al., 2015; ZHANG et al., 2010).

No presente estudo, $P$. stratiotes não sobreviveu na presença do lixiviado proveniente do tratamento convencional sem diluição, o mesmo que foi aplicado no sistema. Isso demonstra que com o decorrer do tempo a espécie poderá ter os valores de cobertura vegetal reduzidos ou até mesmo desaparecer das áreas alagadas naturais, o que diminuirá sua representatividade no pós-tratamento do lixiviado.

Os resultados obtidos atendem à legislação ambiental vigente, constante na Resolução do Conselho Estadual de Meio Ambiente (CEMA) $n^{\circ}$ 86/2013 (PARANÁ, 2013), que estabelece diretrizes e critérios orientadores para o licenciamento e outorga, projeto, implantação, operação e encerramento de aterros sanitários e visa ao controle da poluição, da contaminação e a minimização dos impactos ambientais. O sistema apresentou eficiência de 72 e 30\% na redução de DBO e DQO, respectivamente. Em áreas alagadas, as macrófitas podem reduzir os valores de DBO e DQO por meio da rizodegradação. Nesse processo as macrófitas liberam oxigênio ou exsudados das raízes para a rizosfera, o que estimula o desenvolvimento de micro-organismos que degradam a matéria orgânica (ANDRADE et al. 2007; BARTUCCA et al., 2016; BRIX, 1997; JONES et al., 2006; PREUSSLER et al., 2015; SUSARLA et al., 2002). Os compostos orgânicos também podem ser sequestrados, degradados,

Tabela 2 - Valores da média e desvio padrão das variáveis avaliadas nos três pontos de coleta e a eficiência do pós-tratamento em cada caixa e no sistema.

\begin{tabular}{|c|c|c|c|c|c|c|}
\hline \multirow{2}{*}{ Variáveis } & \multirow{2}{*}{ Ponto $1^{*}$} & \multirow{2}{*}{ Ponto 2} & \multirow{2}{*}{ Ponto 3} & \multicolumn{3}{|c|}{ Eficiência do pós-tratamento (\%) } \\
\hline & & & & 1a caixa & $2^{\mathrm{a}}$ caixa & Sistema piloto \\
\hline $\mathrm{pH}$ & $8,22 \pm 0,22$ & $8,11 \pm 0,02$ & $7,79 \pm 0,01$ & - & - & - \\
\hline $\mathrm{DBO}\left(\mathrm{mg} \cdot \mathrm{O}_{2} \cdot \mathrm{L}^{-1}\right)$ & $213,00 \pm 0,00$ & $31,00 \pm 0,00$ & $59,00 \pm 0,00$ & 85,45 & ** & 72,30 \\
\hline $\mathrm{DQO}\left(\mathrm{mg} \cdot \mathrm{O}_{2} \mathrm{~L}^{-\mathrm{L}^{-1}}\right)$ & $1.888,33 \pm 21,94$ & $939,33 \pm 21,78$ & $1.313,67 \pm 15,82$ & 50,26 & ** & 30,44 \\
\hline P total (mg. L'1) $^{-1}$ & $12,32 \pm 0,91$ & $3,66 \pm 0,04$ & $3,00 \pm 0,04$ & 70,27 & 18,03 & 75,78 \\
\hline $\mathrm{P}-\mathrm{PO}_{4}\left(\mathrm{mg} \cdot \mathrm{L}^{-1}\right)$ & $37,79 \pm 2,79$ & $11,23 \pm 0,12$ & $9,19 \pm 0,11$ & 70,28 & 18,17 & 75,78 \\
\hline Nitrato (mg. $\left.L^{-1}\right)$ & $1.481,82 \pm 30,79$ & $1.071,25 \pm 15,91$ & $917,40 \pm 1,36$ & 27,71 & 14,36 & 38,09 \\
\hline Nitrito (mg. $\left.\mathrm{L}^{-1}\right)$ & $1.042,75 \pm 1,83$ & $468,28 \pm 3,81$ & $702,72 \pm 1,84$ & 55,09 & ** & 32,61 \\
\hline $\mathrm{N}-\mathrm{NH}_{4}\left(\mathrm{mg} \cdot \mathrm{L}^{-1}\right)$ & $670,11 \pm 3,66$ & $199,33 \pm 5,83$ & $282,86 \pm 8,57$ & 70,25 & ** & 57,79 \\
\hline $\mathrm{N}-\operatorname{Norg}\left(\mathrm{mg} \cdot \mathrm{L}^{-1}\right)$ & $94,87 \pm 11,28$ & $24,66 \pm 6,52$ & $43,47 \pm 11,67$ & 74,01 & ** & 43,64 \\
\hline $\mathrm{Cd}\left(\mathrm{mg} \cdot \mathrm{L}^{-1}\right)$ & ND & ND & ND & - & - & - \\
\hline $\mathrm{Cu}\left(\mathrm{mg} \cdot \mathrm{L}^{-1}\right)$ & ND & ND & ND & - & - & - \\
\hline $\mathrm{Ni}\left(\mathrm{mg} . \mathrm{L}^{-1}\right)$ & $1,55 \pm 0,10$ & $1,73 \pm 0,20$ & $3,01 \pm 0,45$ & ** & ** & $* *$ \\
\hline $\mathrm{Zn}\left(\mathrm{mg} \cdot \mathrm{L}^{-1}\right)$ & $0,84 \pm 0,08$ & $0,48 \pm 0,07$ & $0,73 \pm 0,11$ & 42,85 & ** & 13,09 \\
\hline
\end{tabular}

*Ponto 1 refere-se ao lixiviado proveniente do sistema de tratamento convencional sem diluição; **não houve remoção; DBO: demanda bioquímica de oxigênio; DQO: demanda química de oxigênio; P total: fósforo total; $\mathrm{P}-\mathrm{PO}_{4}$ : fósforo inorgânico; N-NH detectado. Limites detecção: Cd (0,003 $\left.\mu \mathrm{g} . \mathrm{g}^{-1}\right)$; Cu (O,16 $\left.\mu \mathrm{g} \cdot \mathrm{g}^{-1}\right)$; Ni $\left(0,17 \mu \mathrm{g} \cdot \mathrm{g}^{-1}\right)$; e Zn $\left(0,11 \mu \mathrm{g} \cdot \mathrm{g}^{-1}\right)$. 
usados no crescimento ou transportados para a parte área das plantas (JONES et al., 2006; PREUSSLER et al., 2015).

$\mathrm{Na}$ remoção de $\mathrm{N}$, o sistema apresentou eficiência de 38\% para nitrato, $32 \%$ para nitrito, $57 \%$ para $\mathrm{N}_{-} \mathrm{NH}_{4}$ e $36 \%$ para N-NOrg. De acordo com Vymazal (2007), a remoção de $\mathrm{N}$ em áreas alagadas está relacionada às transformações que ocorrem durante o ciclo biogeoquímico desse elemento. Sawaittayothin e Polprasert (2007), no tratamento de lixiviado em áreas alagadas construídas, atingiram remoção de $90 \%$ do $\mathrm{N}$ total por meio da captura pelas plantas, nitrificação, desnitrificação e pela volatilização.

$\mathrm{Na}$ volatilização o íon amônio $\left(\mathrm{NH}_{4}^{+}\right)$é convertido na forma gasosa $\left(\mathrm{NH}_{3}\right)$, no entanto, esse processo só é significativo em $\mathrm{pH}$ acima de 9,3 (VYMAZAL, 2007). O pH no sistema piloto, variou de 7,79 a 8,22, portanto, a volatilização não foi relevante.
A captura de $\mathrm{N}$ pelas macrófitas é realizada pela assimilação de $\mathrm{N}-\mathrm{NH}_{4}$ ou nitrato, os quais são convertidos em compostos orgânicos que são utilizados na construção de células e tecidos (BELL et al., 2015; VYMAZAL, 2007). As macrófitas podem auxiliar na remoção de $\mathrm{N}$, pela estratégia de rizodegradação, estimulando a atividade de bactérias nitrificantes e desnitrificantes (ANDRADE et al., 2007; BRIX, 1997; JONES et al., 2006; SUSARLA et al., 2002). As bactérias nitrificantes convertem o N-NH${ }_{4}^{+}$em nitrato e as desnitrificantes transformam o nitrato em $\mathrm{N}_{2}$ (dinitrogênio), em ambos os casos é formado nitrito como composto intermediário (BELL et al., 2015; VYMAZAL, 2007).

Apóstolo (2005), em estudo sobre a anatomia da vegetação costeira do rio Salado, na Argentina, observou que as raízes de Echinochloa crus-gali e outras espécies da família Poaceae apresentam alta proporção de aerênquima, tecido que armazena oxigênio. Preussler et al. (2015) demonstraram

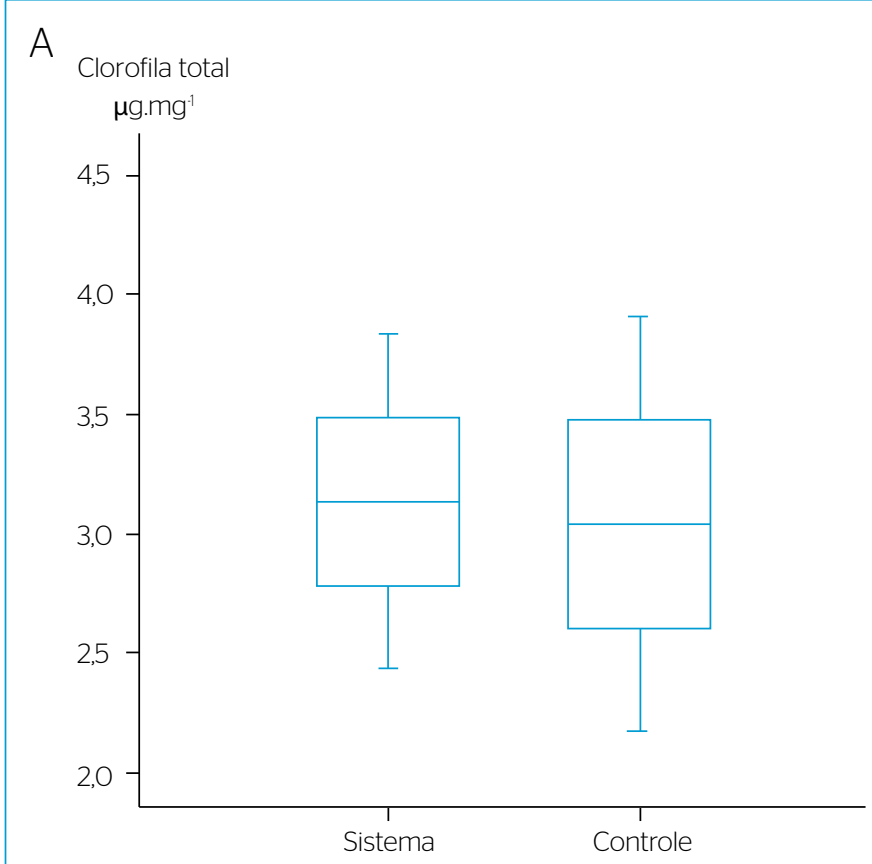

B

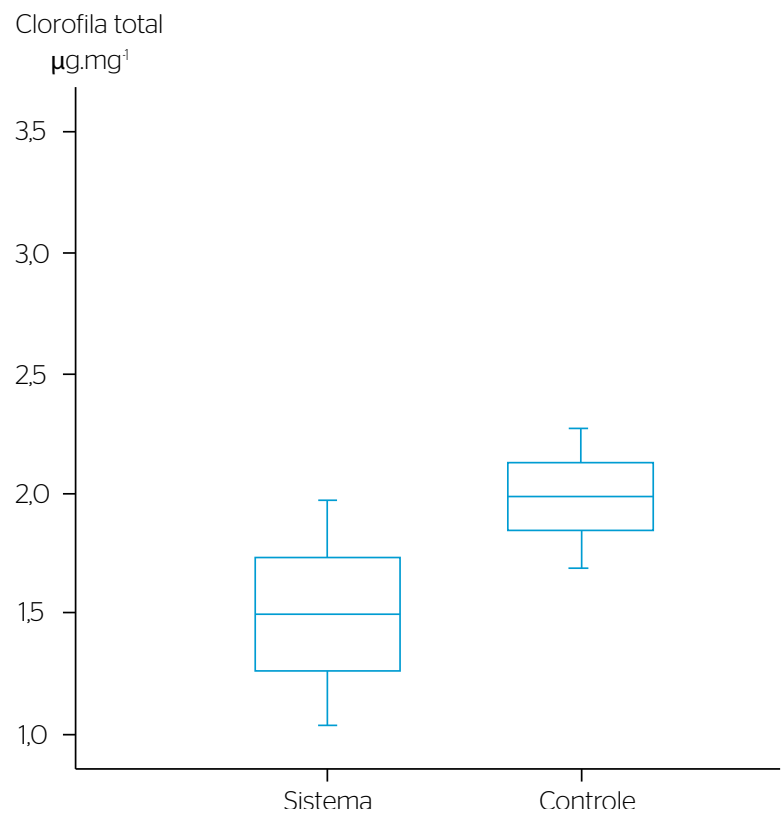

Figura 2 - Médias e desvios padrão do teor de clorofila total das plantas coletadas no sistema e plantas controle. (A) Echinochloa polystachya: (B) Eichhornia crassipes.

Tabela 3 - Valores de média e desvio padrão das concentrações de Cd, Cu, Ni e Zn na parte aérea e na raiz de Echinochloa polystachya e Eichhornia crassipes no sistema e no controle.

\begin{tabular}{|c|c|c|c|c|c|}
\hline \multirow{2}{*}{ Macrófita } & \multirow{2}{*}{ Metais $\left(\mu \mathrm{g} . \mathrm{g}^{-1}\right)$} & \multicolumn{2}{|c|}{ Parte aérea } & \multicolumn{2}{|c|}{ Raiz } \\
\hline & & Caixa & Controle & Caixa & Controle \\
\hline \multirow{4}{*}{$\begin{array}{l}\text { Echinochloa } \\
\text { polystachya }\end{array}$} & $\mathrm{Cd}$ & $4,96 \pm 1,11$ & $2,33 \pm 0,67$ & $3,58 \pm 0,99$ & $3,20 \pm 1,28$ \\
\hline & $\mathrm{Cu}$ & $N D^{*}$ & $N D^{*}$ & $N D^{*}$ & $N D^{*}$ \\
\hline & $\mathrm{Ni}$ & $46,26 \pm 7,48$ & $57,94 \pm 24,28$ & $73,75 \pm 2,77$ & $29,01 \pm 4,98$ \\
\hline & $\mathrm{Zn}$ & $70,56 \pm 5,57$ & $55,95 \pm 7,94$ & $40,31 \pm 5,89$ & $47,65 \pm 7,18$ \\
\hline \multirow{4}{*}{$\begin{array}{l}\text { Eichhornia } \\
\text { crassipes }\end{array}$} & $\mathrm{Cd}$ & $8,45 \pm 0,67$ & $6,36 \pm 0,50$ & $10,19 \pm 1,36$ & $6,77 \pm 0,76$ \\
\hline & $\mathrm{Cu}$ & $15,17 \pm 2,87$ & $N D^{*}$ & $21,94 \pm 4,86$ & $15,77 \pm 2,13$ \\
\hline & $\mathrm{Ni}$ & $66,92 \pm 9,04$ & $53,27 \pm 24,96$ & $50,0 \pm 0,30$ & $56,50 \pm 7,55$ \\
\hline & $\mathrm{Zn}$ & $102,97 \pm 9,93$ & $63,27 \pm 8,75$ & $107,01 \pm 12,12$ & $57,46 \pm 3,40$ \\
\hline
\end{tabular}

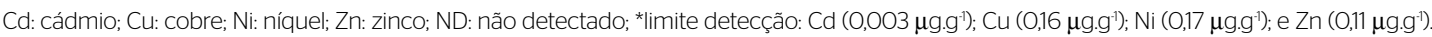


que cerca de 75\% da área total das raízes de Echinochloa polystachya estão representadas por aerênquima.

A presença desse tecido em E. polystachya sugere que a redução das concentrações de matéria orgânica, N e P ocorre por meio da rizodegradação, pois a presença do aerênquima, segundo Yamauchi et al. (2013) e Preussler et al. (2015), permite a tolerância e sobrevivência das macrófitas em ambientes que apresentam condições desfavoráveis e/ou anóxicos. Além disso, essas plantas disponibilizam o oxigênio, armazenado no aerênquima, para o meio, por isso há o favorecimento de condições aeróbicas na região rizosférica das macrófitas, o que estimula o crescimento de micro-organismos que contribuem para a remoção dos poluentes (BARTUCCA et al., 2016; BIALOWIEC et al., 2014; HEADLEY et al., 2012; WANG et al., 2014). Bell et al. (2015) constataram que as macrófitas apresentam a capacidade de assimilar compostos de $\mathrm{N}$ que são desnitrificados a partir da atividade dos micro-organismos presentes na rizosfera, fator que contribui para o maior crescimento e desenvolvimento dessas plantas no ambiente.

O sistema apresentou eficiência de $76 \%$ na redução de fósforo total ( $\mathrm{P}$ total) e $\mathrm{P}-\mathrm{PO}_{4}$. A remoção de $\mathrm{P}$ em áreas alagadas ocorre principalmente por meio da assimilação nos tecidos das plantas, precipitação química com metais, adsorção de fosfato no substrato e ação bacteriana (VYMAZAL, 2007).

Não houve remoção de Ni no sistema e a eficiência do tratamento de Zn foi de $13 \%$. Os metais, nas áreas alagadas, podem ser removidos por meio de adsorção, absorção, precipitação, sedimentação e captura pelas plantas (WEIS; WEIS, 2004). Além disso, as macrófitas podem extrair os metais presentes na água e no sedimento e acumular nos tecidos, processos denominados de fitoextração e fitoacumulação (SUSARLA et al., 2002).

Mannarino et al. (2006), em uma área alagada construída plantada com Typha angustifolia, encontraram bons resultados na remoção de matéria orgânica ( $41 \%$ de DQO e 57\% de DBO), N-NH ( $_{4}(51 \%)$ e sólidos (60\%). Bulc (2006), ao analisar um sistema construído com áreas alagadas de fluxo vertical e horizontal com Phragmites australis e Typha latifolia, encontrou 50\% de eficiência na redução de DQO, 59\% de DBO, $51 \%$ de $\mathrm{N}_{-} \mathrm{NH}_{4}$ e $53 \%$ de $\mathrm{P}$ total. Segundo esse autor a eficiência na redução desses parâmetros está relacionada com a diluição e a concentração do lixiviado, podendo aumentar com a precipitação.

Jayaweera e Kasturiarachchi (2004), no tratamento de efluente industrial com E. crassipes, obtiveram eficiência de $100 \%$ na remoção de $\mathrm{N}$ total e P total. Zimmels et al. (2006), em experimento piloto para o tratamento de esgoto doméstico, relataram que um sistema com as espécies E. crassipes e $P$. stratiotes reduziu e manteve a concentração de DQO e DBO em níveis baixos, 50 a 85 e 4 a 6 mg.L ${ }^{-1}$, respectivamente.

A dosagem de clorofila demonstra que não houve diferença significativa no desenvolvimento de E. polystachya no controle e no sistema. Para o desenvolvimento, as plantas necessitam absorver $\mathrm{N}$ e $\mathrm{P}$, o que pode estar associado à redução no valor de concentração de nitrato, $\mathrm{N}-\mathrm{NH}_{4}, \mathrm{P}$ total e $\mathrm{P}-\mathrm{PO}_{4}$.
Echinochloa polystachya apresentou 43\% de eficiência no tratamento de $\mathrm{Zn}$. O aumento significativo de $\mathrm{Zn}$ na biomassa sugere que a macrófita realizou a fitoextração desse metal. No entanto, os níveis desse elemento estão dentro dos limites de valores para as plantas, de $1 \mathrm{a} 40 \mu \mathrm{g} \cdot \mathrm{g}^{-1}$ (LARCHER, 2000). Essa mesma espécie apresentou 4,96 $\mu \mathrm{g} . \mathrm{g}^{-1} \mathrm{de} \mathrm{Cd}$ na parte aérea e 3,58 $\mu \mathrm{g} \cdot \mathrm{g}^{-1}$ nas raízes, valores mais altos do que o limite para plantas, de 0,05 a 0,2 $\mu \mathrm{g} . \mathrm{g}^{-1}$ (KABATA-PENDIAS; PENDIAS, 2000), mas não tanto como o observado por Solís-Domínguez et al. (2007). Segundo esses autores, a espécie é capaz de armazenar, em suas raízes e folhas, aproximadamente 299 e $233 \mu \mathrm{g} . \mathrm{g}^{-1}$ de Cd, respectivamente, sendo considerada uma planta hiperacumuladora de Cd.

A baixa eficiência de E. crassipes no sistema pode estar relacionada à alta concentração de $\mathrm{N}-\mathrm{NH}_{4}^{+} \mathrm{e}$ ao fato dessa macrófita apresentar sinais de injúria, como clorose, comprovada pelo baixo teor de clorofila total encontrado. Petrucio e Esteves (2000) relatam que E. crassipes apresenta alta eficiência para a absorção de nitrogênio, entretanto, quando esse elemento está presente em altas concentrações, a taxa de absorção é reduzida. Jampeetong e Brix (2009) citam que um dos sintomas de fitoxicidade de $\mathrm{N}-\mathrm{NH}_{4}$ inclui a presença de clorose nas folhas.

Com isso, alguns exemplares de E. crassipes se tornaram senescentes e os poluentes extraídos retornaram ao sistema. A baixa temperatura atingida (mínima de $9,4^{\circ} \mathrm{C}$ ) no período em que E. crassipes ficou na segunda caixa pode explicar os sinais de injúria. Segundo Wilson et al. (2005), a espécie suporta grandes variações de temperatura (1 a $40^{\circ} \mathrm{C}$ ), mas apresenta sensibilidade ao frio.

A presença de metais no lixiviado pode ser um fator que contribuiu para os sinais de injúria em E. crassipes. As concentrações de Cd na parte aérea e nas raízes de $E$. crassipes no controle e no sistema foram maiores do que os valores limites para plantas, de 0,05 a $0,2 \mu \mathrm{g} . \mathrm{g}^{-1}$ (SUSARLA et al., 2002). Segundo Jiang et al. (2005), um dos sintomas visíveis de toxicidade ao Cd é a clorose foliar.

Segundo Liao e Chang (2004) e Malik (2007), com o aumento da concentração de metais, a eficiência de remoção desses por E. crassipes se eleva, mas ao mesmo tempo surgem sintomas de toxicidade, como a inibição da síntese de clorofila e necrose. Por isso, efluentes com alta concentração de metais precisam ser diluídos antes de serem tratados. Em estudo realizado por Silva et al. (2005), E. crassipes não sobreviveu em contato direto com o lixiviado e os autores recomendam realizar uma adaptação gradual dessa macrófita em diferentes concentrações, dado que corrobora o presente trabalho.

\section{CONCLUSÕES}

O pós-tratamento do lixiviado no sistema piloto apresentou eficiência para remoção de DBO, DQO, $\mathrm{P}$ total, $\mathrm{P}-\mathrm{PO}_{4}$, nitrato, nitrito, $\mathrm{N}-\mathrm{NH}_{4}^{+}$, N-NOrg e Zn, atendendo, como pós-tratamento, à legislação ambiental vigente no estado do Paraná. Sugere-se a continuação do presente 
estudo por um período de tempo mais longo para que se possa analisar o crescimento e o desenvolvimento das macrófitas e, com isso, obter informações sobre o manejo em áreas alagadas; o estudo da rizosfera das macrófitas para a compreensão da interação planta-micro-organismo no processo de rizodegradação e sua contribuição para a fitorremediação de livxiviado gerado em aterro sanitário.

\section{REFERÊNCIAS}

AMERICAN PUBLIC HEALTH ASSOCIATION - APHA; AMERICAN WATER WORKS ASSOCIATION -AWWA; WATER ENVIRONMENT FEDERATION - WEF (2005) Standard Methods for the Examination of Water and Wastewater. 21. ed. Washington, D.C.: APHA-AWWA-WEF.

ANDRADE, J.C.M.; TAVARES, S.R.L.; MAHLER, C.F. (2007) Fitorremediação: o uso de plantas na melhoria da qualidade ambiental. São Paulo: Oficina de Textos. 176 p.

APÓSTOLO, N.M. (2005) Caracteres anatómicos de la vegetación costera del Río Salado (Noroeste de la provincia de Buenos Aires, Argentina). Boletín de la Sociedad Argentina de Botánica, v. 40, n. 3-4, p. 215-227.

BARNES, J.D.; BALAGUER, L.; MANRIQUE, E.; ELVIRA, S.; DAVISON, A.W. (1992) A reappraisal of use of DMSO for extraction and determination of chlorophylls a and $\mathrm{b}$ in lichens and higher plants. Environmental and Experimental Botany, v. 32, p. 85-100. https://doi. org/10.1016/0098-8472(92)90034-Y

BARTUCCA, M.L.; MIMMO, T.; CESCO, S.; BUONO, D.D. (2O16) Nitrate Removal from polluted water by using a vegetated floating system. Science and Total Environmental, v. 542, p. 803-808. https://doi. org/10.1016/j.scitotenv.2015.10.156

BELL, C.W.; ASAO, S.; CALDERON, F.; WOLK, B.M.; WALLENSTEIN, D. (2015)Plantnitrogenuptakedrives rhizospherebacterialcommunity assembly during plant growth. Soil Biology \& Biochemistry, v. 85, p. 170-182. https://doi.org/ 10.1016/j.soilbio.2015.03.006

BERTAZZOLI, R.; PELEGRINI, R. (2002) Descoloração e degradação de poluentes orgânicos em soluções aquosas através do processo fotoeletroquímico. Química Nova, São Paulo, v. 25, n. 3, p. 470-476. http://dx.doi.org/10.1590/S0100-40422002000300022

BIALOWIEC, A.; ALBUQUERQUE, A.; RANDERSON, P.F. (2014) The influence of evapotranspiration on vertical flow subsurface constructed wetland performance. Ecological Engineering, v. 67, p. 89-94. https://doi.org/10.1016/j.ecoleng.2014.03.032

BRAUN-BLANQUET, J. (1979) Fitossociologia: base para el estúdio de las comunidades vegetales. Madri: H. Blume Ediciones. 820 p.

BRIX, H. (1994) Functions of macrophytes in constructed wetlands. Water Science and Technology, v. 29, n. 4, p. 71-78.

(1997) Do macrophytes play a role in constructed wetlands? Water Science and Technology, v. 35, n. 5, p. 11-17. https://doi. org/10.1016/SO273-1223(97)00047-4
BULC, T.G. (2006) Long term performance of a constructed wetland for landfill leachate treatment. Ecological Engineering. v. 26, p. 365-374. https://doi.org/10.1016/j.ecoleng.2006.01.003

CARNEIRO, C.; REISSMAMN, C.B.; MARQUES, R. (2006) Comparação de métodos de análise química de K, Ca, Mg e Al em folhas de ervamate (/lex paraguarienses, St. Hil.). Cerne, Lavras, v. 12, n. 2, p. 113-122.

CARVALHO, A.B.P.; OZORIO, C.P. (2007) Avaliação sobre os banhados do Rio Grande do Sul, Brasil. Revista de Ciências Ambientais, Canoas, v. 1, n. 2, p. 83-85. http://dx.doi.org/10.18316/171

CASTILHOS JR., A. B.; DALSASSO, R. L.; ROHERS, F. (2010) Prétratamento de lixiviados de aterros sanitários por filtração direta ascendente e coluna de carvão ativado. Revista Engenharia Sanitária e Ambiental, Rio de Janeiro, v. 15, n. 4, p. 385-392.

CELERE, M.S.; OLIVEIRA, A.S.; TREVILATO, T.M.B.; SEGURA-MUÑOZ, S. I. (2007) Metais presentes no chorume coletado no aterro sanitário de Ribeirão Preto, São Paulo, Brasil, e sua relevância para saúde pública. Cadernos de Saúde Pública, v. 23, n. 4, p. 939-947. http://dx.doi.org/10.1590/S0102-311X2007000400021

COMPANHIA DE TECNOLOGIA DE SANEAMENTO AMBIENTAL CETESB. (2004) Manual de Espectrometria de Absorção Atômica CHAMA. São Paulo: CETESB.

ENVIRONMENTAL PROTECTION AGENCY - U. S. EPA. (1988) Design Manual: Constructed Wetlands and Aquatic Plant Systems for Municipal Wastewater Treatment. Cincinnati: U. S. EPA. 83 p.

(1998) Method 3050 B. Cincinnati: U. S. EPA.

GRANATO, M. (1995) Utilização do aguapé no tratamento de efluentes com cianetos. Rio de Janeiro: CETEM/CNPq. 39 p.

HEADLEY, T.R.; DAVISON, L.; HUETT, D.O; MULLER, E. (2O12) Evapotranspiration from subsurfaces horizontal flow wetlands planted with Phragmites australis in sub-tropical Austrália. Water Research, v. 46, p. 345-354. https://doi.org/10.1016/j.watres.2011.10.042

JAMPEETONG, A.; BRIX, H. (2OO9) Effects of NH4+ concentration on growth, morphology and $\mathrm{NH} 4+$ uptake kinetics of Salvinia natans. Ecological Engineering, v. 35, p. 695-702. https://doi. org/10.1016/j.ecoleng.2008.11.006

JAYAWEERA, M.W.; KASTURIARACHCHI, J.C. (2004) Removal of nitrogen and phosphorus from industrial wastewater by phytoremediation using water hyacinth (Eichhornia crassipes (Mart.) Solms.). Water Science and Technology, v. 50, p. 217-225. 
JIANG, R.F; MA, D.Y; ZHAO, F.J.; MCGRATH, S.P. (2005) Cadmium hyper accumulation protects Thlaspi caerulescens from leaf feeding damage by thrips (Frankliniella occidentalis). New Phytologist, v. 167, p. 805-814.

JONES, D.L.; WILLIAMSON, K.L.; OWEN, A.G. (2006) Phytoremediation of landfill leachate. Waste Management, v. 26, n. 8, p. 825-837. https://doi.org/10.1016/j.wasman.2005.06.014

KABATA-PENDIAS, A.; PENDIAS, H. (2000) Trace elements in soils and plants. 3. ed. Boca Raton: CRC Press.

KADLEC, R.H.; KNIGHT, R.I. (1996) Treatment wetlands. Boca Raton: Lewis Publishers. $893 \mathrm{p}$.

KADLEC, R.H.; ZMARTHIE, L.A. (2010) Wetland treatment of leachate from a closed landfill. Ecological Engineering, v. 36, n. 7, p. 946-957. DOI: 10.1016/j.ecoleng.2010.04.013

LAMEGO, F.P.; VIDAL, R.A. (2007) Fitorremediação: plantas como agentes de despoluição? Pesticidas: Revista Ecotoxicologia e Meio Ambiente, Curitiba, v. 17, p. 9-18. http://dx.doi.org/10.5380/pes.v17i0.10662

LARCHER, W. (2000) Ecofisiologia vegetal. São Carlos: RiMa. 531 p.

LIAO, S.W.; CHANG, W.L. (2004) Heavy metal phytoremediation by water hyacinth at constructed wetlands in Taiwan. Journal of Aquatic Plant Management, v. 42, p. 60-68.

MALIK, A. (2007) Environmental challenge vis a vis opportunity: the case of water hyacinth. Environment International, v. 33, p. 122-138. https://doi.org/10.1016/j.envint.2006.08.004

MANNARINO, C.F.; FERREIRA, J.A.; CAMPOS, J.C.; RITTER, E. (2006) Wetlands para tratamento de lixiviados de Aterros Sanitários - Experiências no Aterro Sanitário de Piraí e no Aterro Metropolitano de Gramacho (RJ). Revista Engenharia Sanitária e Ambiental, v. 11, n. 2, p. 108-112.

PARANÁ. Conselho Estadual de Meio Ambiente - CEMA. Resolução CEMA no 86, de 2 de abril de 2013. Diário Oficial da União, n. 8941, 19 abr. 2013. Disponível em: <http://www.legislacao.pr.gov.br/ legislacao/listarAtosAno.do?action=exibir\&codAto=97097\&indice= $1 \&$ totalRegistros=5\&anoSpan=2014\&anoSelecionado=2013\&mesS elecionado=0\&isPaginado=true $>$. Acesso em: 19 nov. 2016.

PETRUCIO, M.M.; ESTEVES, F.A. (2000) Uptake rates of nitrogen and phosphorus in the water by Eichhornia crassipes and Salvinia auriculata. Revista Brasileira de Biologia, v. 60, p. 229-236. http:// dx.doi.org/10.1590/S0034-71082000000200006

POMPÊO, M. (2008) Monitoramento e manejo de macrófitas aquáticas. Oecologia Brasiliensis, Rio de Janeiro, v. 12, n. 3, p. 406-424.

PREUSSLER, K.H.; MAHLER, C.F.; MARANHO, L.T. (2015) Performance of a system of natural wetlands in a leachate of a posttratment landfill. International Journal of Environmental Science and technology, v. 12, p. 2623-2638. https://doi.org/10.1007/s13762-014-0674-0
RENOU, S.; GIVAUDAN, J.G.; POULAIN, S.; DIRASSOUYAN, F.; MOULIN, P. (2008) Landfill leachate treatment: Review and opportunity. Journal of Hazardous Materials, v. 150, n. 3, p. 468-493. https://doi.org/10.1016/j.jhazmat.2007.09.077

SAWAITTAYOTHIN, V.; POLPRASERT, C. (2007) Nitrogen mass balance and microbial analysis of construced wetlands treating municipal landfill leachate. Bioresource Technology, v. 98, p. 565570. https://doi.org/10.1016/j.biortech.2006.02.002

SILVA, S.V.A.; MONTEIRO, V.E.D; PAIVA, W. (2005) Tratamento do chorume através do aguapé (Eichhornia crassipes) no Aterro da Muribeca, Pernambuco.In:CONGRESSOBRASILEIRODEENGENHARIA SANITÁRIA E AMBIENTAL, 23., 2005. Anais... Campo Grande: ABES.

SOLIIS-DOMÍNGUEZ, F.A.; GONZÁLEZ-CHÁVEZ, M.C.; CARRILLOGONZÁLEZ, R.; RODRÍGUEZ-VÁZQUEZ, R. (2007) Accumulation and localization of cadmium in Echinochloa polystachya grown within a hydroponic system. Journal of Hazardous Materials, v. 141, p. 630-636. https://doi.org/10.1016/j.jhazmat.2006.07.014

SUSARLA, S.; MEDINA, V.F.; MCCUTCHEON, S.C. (2002) Phytoremediation: an ecological solution to organic chemical contamination. Ecological Engineering, v. 18, n. 5, p. 647-658. https:// doi.org/10.1016/SO925-8574(02)00026-5

VYMAZAL, J. (2007) Removal of nutrients in various types of constructed wetlands. Science of the Total Environment, v. 380, p. 48-65. https://doi.org/10.1016/j.scitotenv.2006.09.014

WANG, C.; ZHENG, S.; WANG, P.; QIAN J. (2014) Effect of vegetation on the removal of contaminants in aquatic environments: A review. Journal of Hydrodynamics, v. 24, p. 497-511. https://doi.org/10.1016/ S1001-6058(14)60057-3

WEIS, J.; WEIS, P. (2004) Metal uptake, transport and release by wetland plants: implications for phytoremediation and restoration Environment International, v. 30, n. 5, p. 685-700. https://doi. org/10.1016/j.envint.2003.11.002

WILSON, J.R.; HOLST, N.; REES, M. (2005) Determinants and patterns of population growth in water hyacinth. Aquatic Botany, v. 81, n. 1, p. 51-67. https://doi.org/10.1016/j.aquabot.2004.11.002

YAMAUCHI, T.; SHIMAMURA, S.; NAKAZONO, M.; MOCHIZUKI, T. (2013) Aerenchyma formation in crop species: A Review. Field Crops Research, v. 152, p. 8-16. https://doi.org/10.1016/j.fcr.2012.12.008

ZHANG, B.Y.; ZHENG, J.S.; SHARP, R.G. (2O10) Phytoremediation in Engineered Wetlands: Mechanisms and Applications. Procedia Environmental Sciences, v. 2, p. 1315-1325. https://doi.org/10.1016/j. proenv.2010.10.142

ZIMMELS, Y.; KIRZHNER, F.; MALKOVSKAJA, A. (2006) Application of Eichhornia crassipes and Pistia stratiotes for treatment of urban sewage in Israel. Journal of Environmental Management, v. 81, n. 4 , p. 420-428. https://doi.org/10.1016/j.jenvman.2005.11.014 